\title{
LAS ESPECIES Y FORMAS DE DICTYOPHORA (FUNGI, BASIDIOMYCETES, PHALLALES) EN MEXICO Y OBSERVACIONES SOBRE SU DISTRIBUCION EN AMERICA LATINA ${ }^{1}$
}

\author{
Gaston Guzman \\ Leticia Montoya \\ $Y$ \\ VICTOR M. BANDALA \\ Instituto de Ecología \\ Apartado Postal 63 \\ Xalapa, Veracruz 91000
}

\section{RESUMEN}

Se presenta una clave de las especies y formas mexicanas del género Dictyophora, el análisis de su distribución en el país (en 10 entidades federativas) y observaciones sobre su presencia en Guatemala, Costa Rica y Brasil. Se concluye que $D$. duplicata (Bosc) Fischer es de climas templados húmedos y de América del Norte y las 3 formas de $D$. indusiata (Vent.: Pers.) Desv. [D.indusiata f. indusiata, $D$. indusiata f. lutea (Liou \& Hwang) Kobayasi y D. indusiata f. rosea (Cesati) Kobayasi] son tropicales y subtropicales.

\begin{abstract}
A study of the genus Dictyophora is presented, providing a key to the Mexican species and forms, as well as their distributional analysis in the country (through 10 States), and observations on the presence of the genus in Guatemala, Costa Rica and Brazil. In conclusion D. duplicata (Bosc) Fischer is distributed in damp temperate regions and in North America, while the 3 forms of $D$. indusiata (Vent.: Pers.) Desv. $[D$. indusiata f. indusiata, D. indusiata $f$. lutea (Liou \& Hwang) Kobayasi and D. indusiata f. rosea (Cesati) Kobayasi] are widespread in tropical and subtropical regions.
\end{abstract}

\section{INTRODUCCION}

Dictyophora Desvaux es uno de los géneros de los Phallales (Gasteromycetes) bien representados en México, tanto en las tierras calientes (vegetación tropical) como en las templadas (bosques de pinos y encinos). Se caracteriza por su esbeltez (fructificaciones mayores de $10 \mathrm{~cm}$ de alto) y por el llamativo indusio, largo o corto según las especies, así como

1 Contribución apoyada por el CONACYT y el Instituto de Ecología. Una modificación de este trabajo fue presentada en el III Congreso Nacional de Micología en Cd. Victoria, Tamaulipas, en octubre de 1988. 
por el olor más o menos desagradable que presenta todo el hongo, principalmente en la gelatinosa gleba.

D. indusiata (Vent.: Pers.) Desv. es común en los trópicos. En América Latina ha sido registrada del Caribe (Trinidad y Puerto Rico), Guayana Francesa, Panamá y Venezuela (Dennis, 1970; Stevenson, 1975; Guzmán, 1986). Por otra parte, D. merulina Berk. y $D$. multicolor B. \& Br. fueron registradas del Caribe (Trinidad y Tobago) (Reid, 1977). D. duplicata (Bosc) Fisch. se conoce de E.U.A. (Smith, 1951), de Europa (Pilát, 1958) y de zonas templadas de México, como se discutirá más adelante.

El primer registro del género en México tuvo lugar en la década de los 50 , cuando Heim y Wasson dieron a conocer $D$. phalloidea Desv. (= D. indusiata) del Estado de Oaxaca, en relación con el uso de este hongo por parte de los Chinantecos, quienes le atribuyen una "fuerza divinatoria" (Heim y Wasson, 1958; Wasson, 1959; Guzmán, 1959; Kobayasi, 1965b; Guzmán y Herrera, 1973). Desde entonces $D$. indusiata ha sido citada de diversas partes del país por Guzmán (1973, 1977, 1986), Welden y Guzmán (1978), Guzmán-Dávalos y Guzmán (1979), López et al. (1980), Chacón y Guzmán (1984) y Portugal et al. (1985). Por otra parte, $D$. duplicata solamente se conocía del Estado de Zacatecas, según el registro de Acosta y Guzmán (1984).

En el presente trabajo se registra una nueva localidad en México de $D$. duplicata y siguiendo a Kobayasi (1965a, 1965b) se reconocen 3 formas taxonómicas de $D$. indusiatay se analiza la distribución de las mismas en México y en América Latina.

Se estudiaron todas las colecciones de Dictyophora preservadas en los herbarios mexicanos de la Escuela Nacional de Ciencias Biológicas del IPN (ENCB), del Instituto de Botánica de la Universidad de Guadalajara (IBUG), de la Facultad de Ciencias de la UNAM (FCME) y del Instituto de Ecología (XAL); una del Instituto de Historia Natural de Chiapas (CHIP) y algunas colectas efectuadas por los autores y colaboradores y depositadas en ENCB, XAL, XALU e ITCV.

\section{LAS ESPECIES DE DICTYOPHORA CONOCIDAS EN EL PAIS}

Según Kobayasi (1965b), Dictyophora incluye 4 especies: $D$. indusiata, $D$. duplicata, $D$. merulina y $D$. multicolor, las diferencias entre las cuales radican en el tipo de superficie del píleo (en donde se localiza la gleba) y en el tamaño y color del indusio. De acuerdo con las observaciones realizadas por los autores, las esporas son iguales en todas las especies: cilíndricas o alantoides, hialinas o verdosas en $\mathrm{KOH}(5 \%)$, de 3-4 × 1-2 $\mu \mathrm{m}$ (Figs. 1-4). Se presenta a continuación una clave de identificación de las especies y formas conocidas de México, Guatemala, Costa Rica y Brasil.

1a. Indusio corto, no mayor de $35 \mathrm{~mm}$ de longitud. Hongos de bosques templados

D. duplicata

1b. Indusio largo, mayor de $35 \mathrm{~mm}$ de longitud. Hongos de selvas tropicales o bosques subtropicales (D. indusiata)

2a. Indusio blanco D. indusiata f. indusiata

2b. Indusio de otro color 3 
Guzmán, et al.: Especies de Dictyophora en México y su Distribución en América Latina
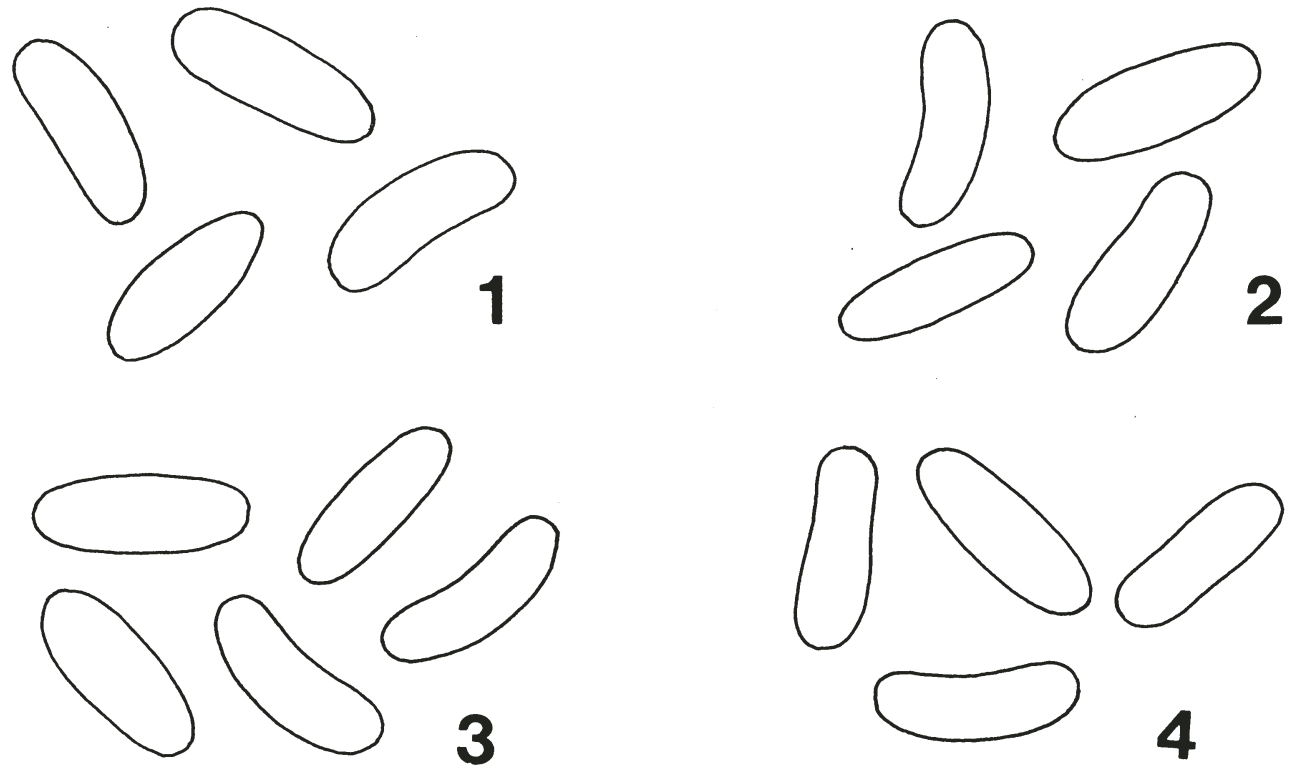

Figs. 1-4. Esporas de Dictyophora. 1. D. duplicata (Acosta 570); 2. D. indusiata f. indusiata (Guzmán 15808); 3. D. indusiata f. lutea (Heredia 447; 4. D. indusiata f. rosea (Pérez-Ortiz 276). 
3a. Indusio amarillo

D. indusiata f. lutea

3b. Indusio de color rosa

D. indusiata f. rosea

\section{Dictyophora duplicata}

D. duplicata (Bosc) Fischer. Ann. Jard. Bot. Buitenz. 6: 23. 1885.

Figs. 1 y 5

Este hongo se caracteriza por su indusio corto y por crecer en zonas templadas de Europa y E.U.A. (Pilát, 1958; Smith, 1951). En México, además de su ya conocida presencia en el Estado de Zacatecas (Acosta y Guzmán, 1984), se presenta ahora el primer registro de Jalisco. En ambos casos se le ha observado en bosques de pino-encino con influencia tropical.

Material revisado. JALISCO: Municipio de Zapopan, La Primavera, Avalos 1 (IBUG). ZACATECAS: $6 \mathrm{~km}$ al NO de Monte Escobedo, Acosta 570 (ENCB).

\section{Dictyophora indusiata}

D. indusiata (Vent.: Pers.) Desvaux. Jour. Bot. 2: 92.1809.

f. indusiata Figs. 2, 5 y 6-7

Esta forma es la más común de las tres, no tan sólo en México, sino a nivel mundial. En América se conoce de México, Guatemala, Panamá, Trinidad, Guayana Francesa y Venezuela. El registro de Guatemala se basa en un ejemplar colectado por Torres y cuya fotografía fue proporcionada a uno de los autores (Guzmán) (Fig. 7); las otras referencias se basan en Dennis $(1953,1970)$, quien al describir $D$. indusiata indicó que el indusio es "white to pale pink", con lo cual indudablemente consideró las formas indusiata y rosea.

Los especímenes mexicanos fueron colectados dentro de los bosques, tanto en la vegetación clímax como en áreas perturbadas e incluso a la orilla de caminos. Las ilustraciones que presentaron Guzmán-Dávalos y Guzmán (1979, pág. 102, fig. 16) y Guzmán (1977, láms. 207 y 213 y 1986, pág. 114, fig. 1) corresponden a esta forma.

Material revisado. CHIAPAS: carretera Ocozocuautla a Malpaso, $\mathrm{km} \mathrm{18.5,} \mathrm{Laguna}$ Bélgica, Cuevas-García 9 (FCME); región de la Selva Lacandona, Municipio de Ocosingo, Chacón s.n., enero 16, 1984 (XAL); Tuxtla Gutiérrez, Jardín Botánico Faustino Miranda, Palacios s.n. (CHIP); alrededores de las ruinas de Palenque, Chacón 3296 (XAL). GUERRERO: Municipio de Mochitlán, Agua de Obispo, Capello 43 (FCME). MORELOS: Municipio de Tepoztlán, zona de El Texcal, camino de Ojo de Agua, Portugal 2 (ENCB). OAXACA: carretera Tuxtepec a Palomares, Rancho El Mirador, Pérez-Ortiz 718 (ENCB); carretera Coatzacoalcos a Salina Cruz, cerca de Palomares, Guzmán 10662 (XALU). QUINTANA ROO: 2 km de la costa, entre Akmal y Xel-ha, Aventuras, Mackinnon s.n., 1978 (XAL, solamente la fotografía a colores). TABASCO: Villahermosa, Parque Museo de La Venta, Mata 54 (XAL). VERACRUZ: Municipio de Atzalan, Arroyo Colorado, Ventura 12575 
Guzmán, et al.: Especies de Dictyophora en México y su Distribución en América Latina

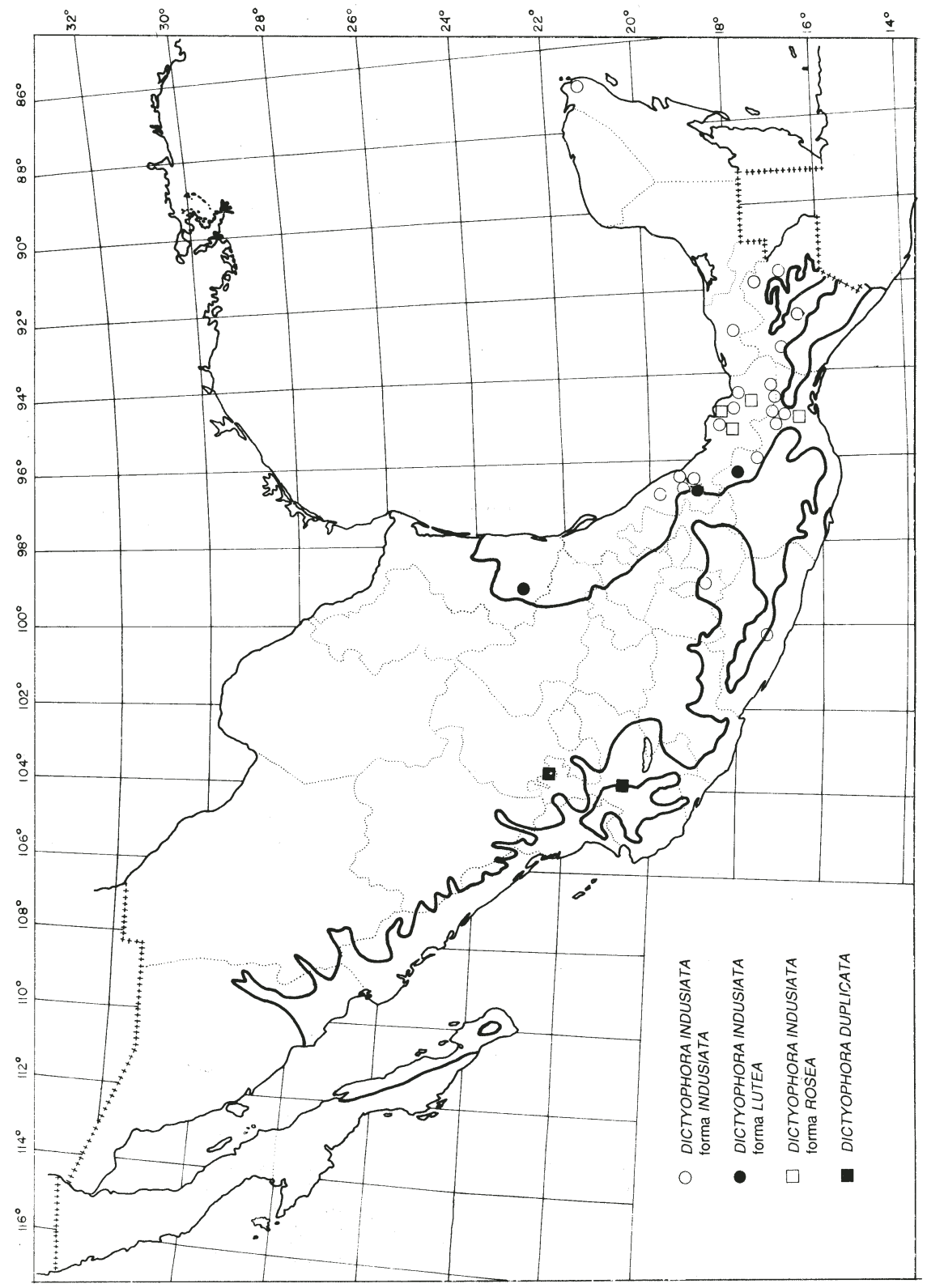

Fig. 5. Distribución conocida de las especies de Dictyophora en México. La línea delimita las tierras calientes (hacia el mar) de las templado-frías (hacia el centro y norte principalmente). Cada punto representa una localidad o varias muy vecinas. 

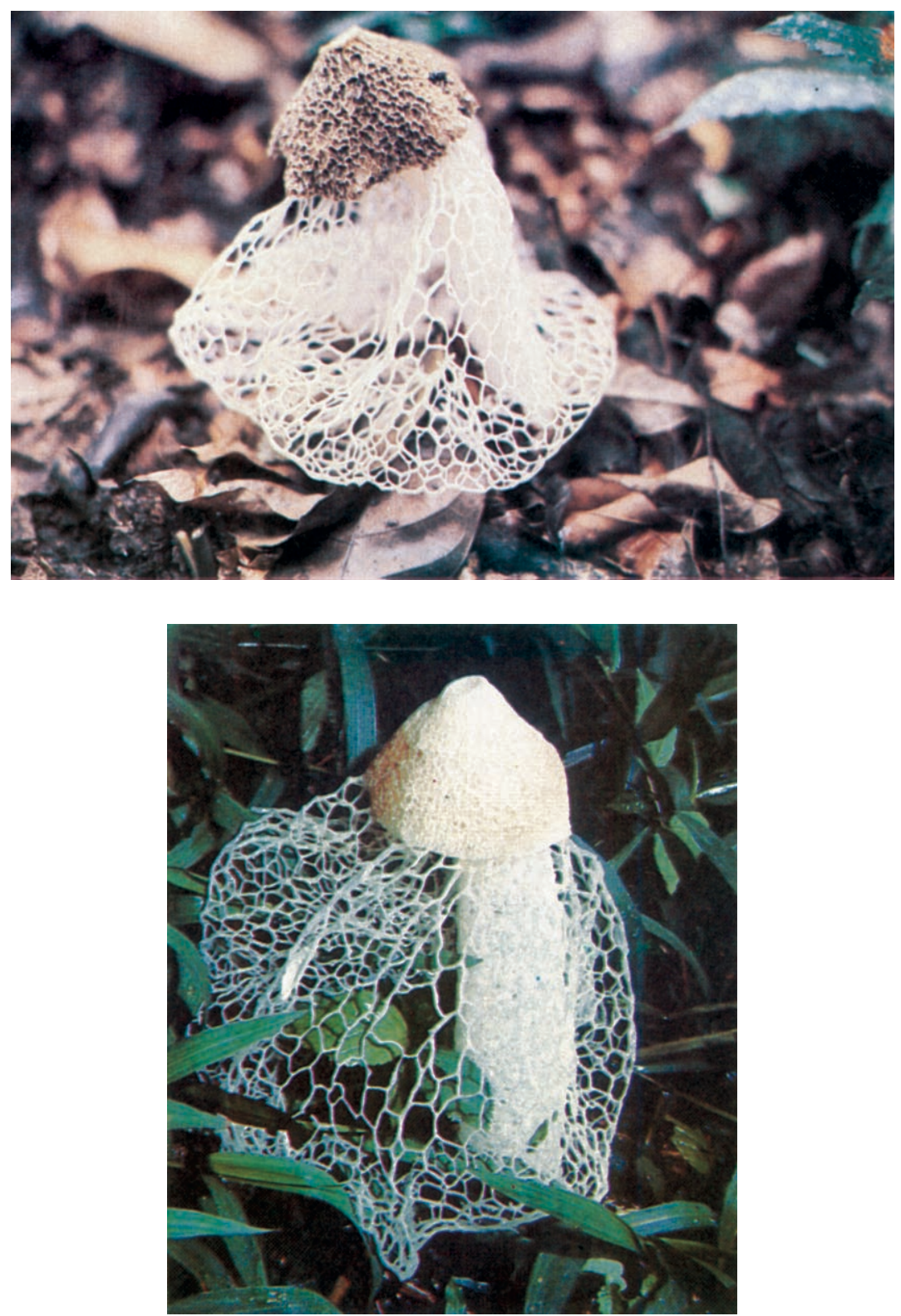

Figs. 6-7 Dictyophora indusiata f. indusiata. 6. Vázquez-Torres s.n. de Uxpanapa, Ver. (foto VázquezTorres). 7. Torres s.n. de Guatemala (foto Torres). Nótese en la Fig. 7 el color rosa del píleo debido a la pérdida de la glebá por la acción de los insectos, la cual todavía se observa en la Fig. 6. 
(ENCB); carretera Paso de Ovejas a Huatusco, km 15, Martínez 44 (XALU); Municipio de Huatusco, Tepezingo, Ventura 11735 (ENCB); Municipio de Actopan, Estación Biológica de La Mancha, Ventura 8909 (ENCB); 3 km al N de Jáltipan, Pérez-Ortiz 395 (ENCB); cerca de Minatitlán, Cosoleacaque, Guzmán 17278 (ENCB); entre Coatzacoalcos y Minatitlán, cerca de Mapachapa, Pérez-Ortiz 177 (ENCB); zona de Uxpanapa, cerca del Campamento Hermanos Cedillo, Vázquez-Torres s.n., junio 20, 1974 (XALU); camino La Laguna al Campamento Uxpanapa, López 605 (ENCB), Guzmán 15808 (ECNB), 16000 (ENCB); región de Los Tuxtlas, camino Catemaco a Montepío, cerca de El Jical, Guzmán 17063 (ENCB).

D. indusiata f. lutea (Liou \& Hwang) Kobayasi. Jour. Jap. Bot. 40: 179. 1965.

Figs. 3,5 y $8-10$

Esta forma solamente se conocía del SE de Asia y se caracteriza por su indusio amarillo, el cual en los materiales herborizados pasa a anaranjado amarillento. Además de los ejemplares de México abajo anotados, se registra también de Brasil (Fig. 9): Rio Grande do Sul, Mpio. de Gravate, Morungava, Guzmán 8820-B (marzo 2, 1971) (ENCB). El peridio de esta forma varía de blanco a color de rosa, según se observó en los materiales estudiados. Por otra parte, Campos (1987) registró a esta variedad (como D. indusiata) de Costa Rica, observación basada en una fotografía a colores que publicó.

Este hongo crece dentro de los bosques tropicales y subtropicales, pero también prospera en áreas perturbadas o cultivadas.

Material revisado. OAXACA: S de Tuxtepec, $3 \mathrm{~km}$ al $S$ del Cerro Sebastopol, Rzedowski s.n., junio 27, 1975 (ENCB). TAMAULIPAS: Municipio de Gómez Farías, Reserva de La Biósfera El Cielo, Sierra Chiquita, Heredia 447 (ENCB, XAL); cerca de la población Gómez Farías, Bandala-Muñoz 1405 (ITCV). VERACRUZ: cerca de Fortín (material de la I Exposición de Hongos de Córdoba), Guzmán 29509 (XAL).

D. indusiata f. rosea (Cesati) Kobayasi. Jour. Jap. Bot. 40: 181. 1985.

Figs. 4,5 y 11

Esta forma se conocía del SE de Asia (Kobayasi, 1965a, 1965b) y de los trópicos americanos (basándose en la descripción de Dennis, 1953,1970, de D. indusiata; ver comentarios en el texto correspondiente a $D$. indusiata $f$. indusiata). Crece preferentemente en lugares perturbados de los bosques tropicales.

Material revisado. OAXACA: carretera Coatzacoalcos a Salina Cruz, cerca de Matías Romero, Guzmán 15713-B (ENCB). VERACRUZ: carretera Minatitlán a Acayucan, NE de Cosoleacaque, Pérez-Ortiz 276 (ENCB); Estación Biológica de Los Tuxtlas, UNAM, Chacón 3249 (XAL); camino El Jical a la Estación Biológica de Los Tuxtlas, Guzmán 19727 (XAL). 


\section{DISCUSION SOBRE LA DISTRIBUCION DE LAS ESPECIES Y FORMAS EN MEXICO}

Analizando la distribución de los materiales mexicanos estudiados, la cual se resume en la figura 5 y la información bibliográfica (Coker y Couch, 1928; Pilát, 1958; Kobayasi, 1965a, 1965b; Smith, 1951; Dring y Rayner, 1967), puede concluirse que $D$. duplicata es una especie de clima templado húmedo, común en los bosques del este de E.U.A., del centro de Europa y de Asia. En México está asociada exclusivamente a los bosques de pino-encino septentrionales con influencia tropical y solamente se conoce de cañadas con este tipo de vegetación en los Estados de Jalisco y Zacatecas. Es probable que tenga una distribución más amplia en los Estados norteños del país.

Referente a $D$. indusiata, las 3 formas existentes en México están confinadas a la vegetación tropical, tanto al bosque tropical perennifolio como al tropical caducifolio, aunque la f. indusiata extiende su distribución con baja frecuencia a los bosques subtropicales (mesófilo de montaña o de pino-encino con influencia tropical, como son los casos observados en las colectas de Fortín y Huatusco en Veracruz y en la de Guerrero).

No parece existir ningún patrón que marque las particularidades de distribución en las 3 formas de $D$. indusiata, aunque es de hacerse notar que la f. lutea presenta el caso de encontrarse en la localidad tropical más septentrional del país y a su vez se conoce del sur de Brasil (Rio Grande do Sul), en donde domina la vegetación subtropical y además Kobayasi (1965a) la circunscribió al extremo norte del área sur-asiática.

\section{AGRADECIMIENTOS}

Los autores hacen patente su agradecimiento al Consejo Nacional de Ciencia y Tecnología y al Instituto de Ecología el apoyo brindado a sus investigaciones. También reconocen la colaboración de los encargados de los herbarios, Biólogos Laura GuzmánDávalos del IBUG, Joaquín Cifuentes del FCME, Jesús García del ITCV y Ricardo Valenzuela del ENCB, por las facilidades brindadas. Se dan las gracias a la Bióloga Gabriela Heredia y al Téc. Arturo Trejo, del Instituto de Ecología, por facilitar material de herbario de Tamaulipas y elaborar el mapa aquí presentado, respectivamente. Uno de los autores (Guzmán) agradece al Biól. E. Palacios, Jefe del Departamento de Botánica del Instituto de Historia Natural de Chiapas, la información y facilidades brindadas. Asimismo reconocen al SNI el apoyo a sus investigaciones. 
Guzmán, et al.: Especies de Dictyophora en México y su Distribución en América Latina
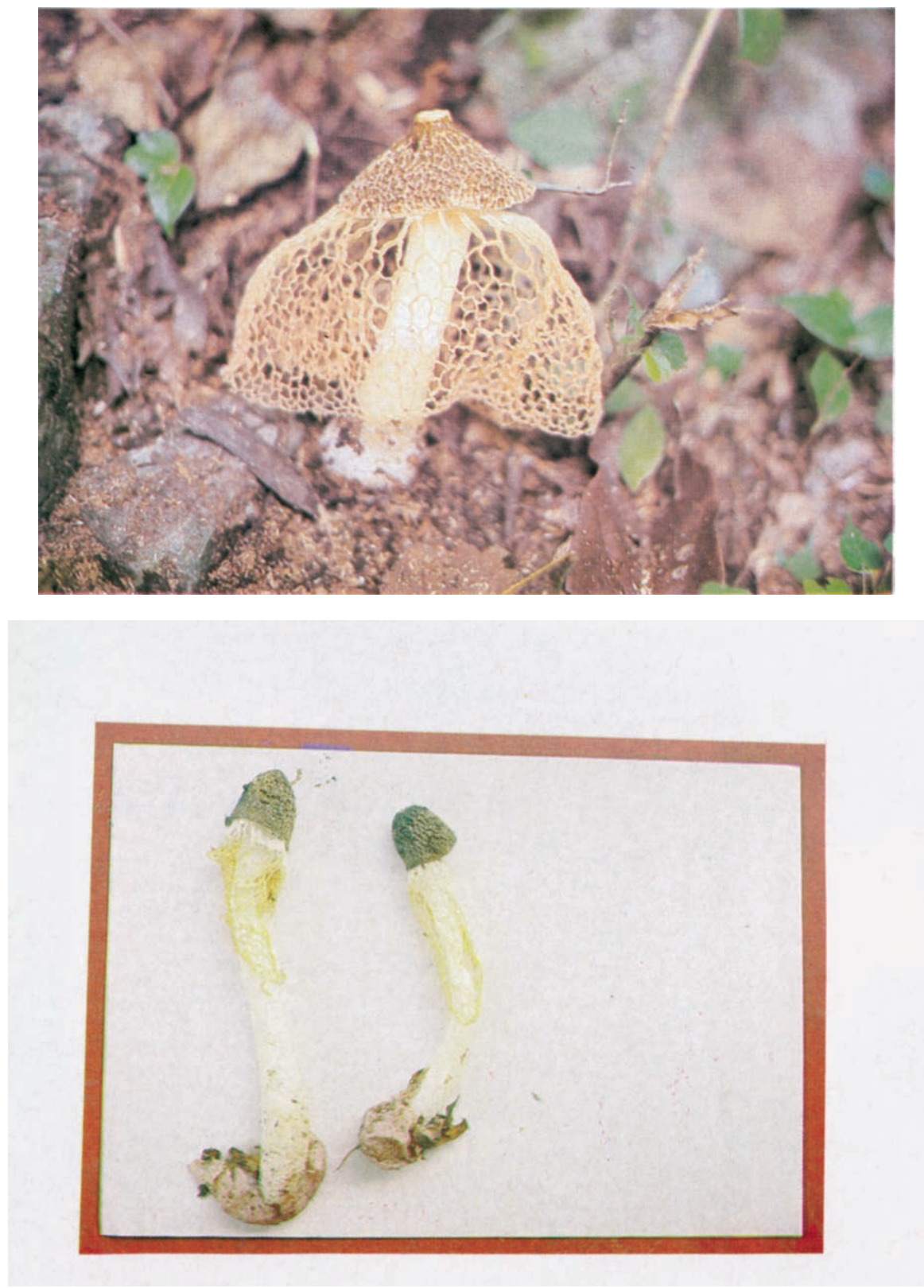

Figs. 8-9 Dictyophora indusiata f. lutea. 8. Heredia 447 de Tamaulipas (foto Heredia). 9. Guzmán 8820$B$ de Brasil (foto Guzmán). En los ejemplares de Brasil nótese el peridio de color rosa, no asi en los de Tamaulipas que es blanco. 

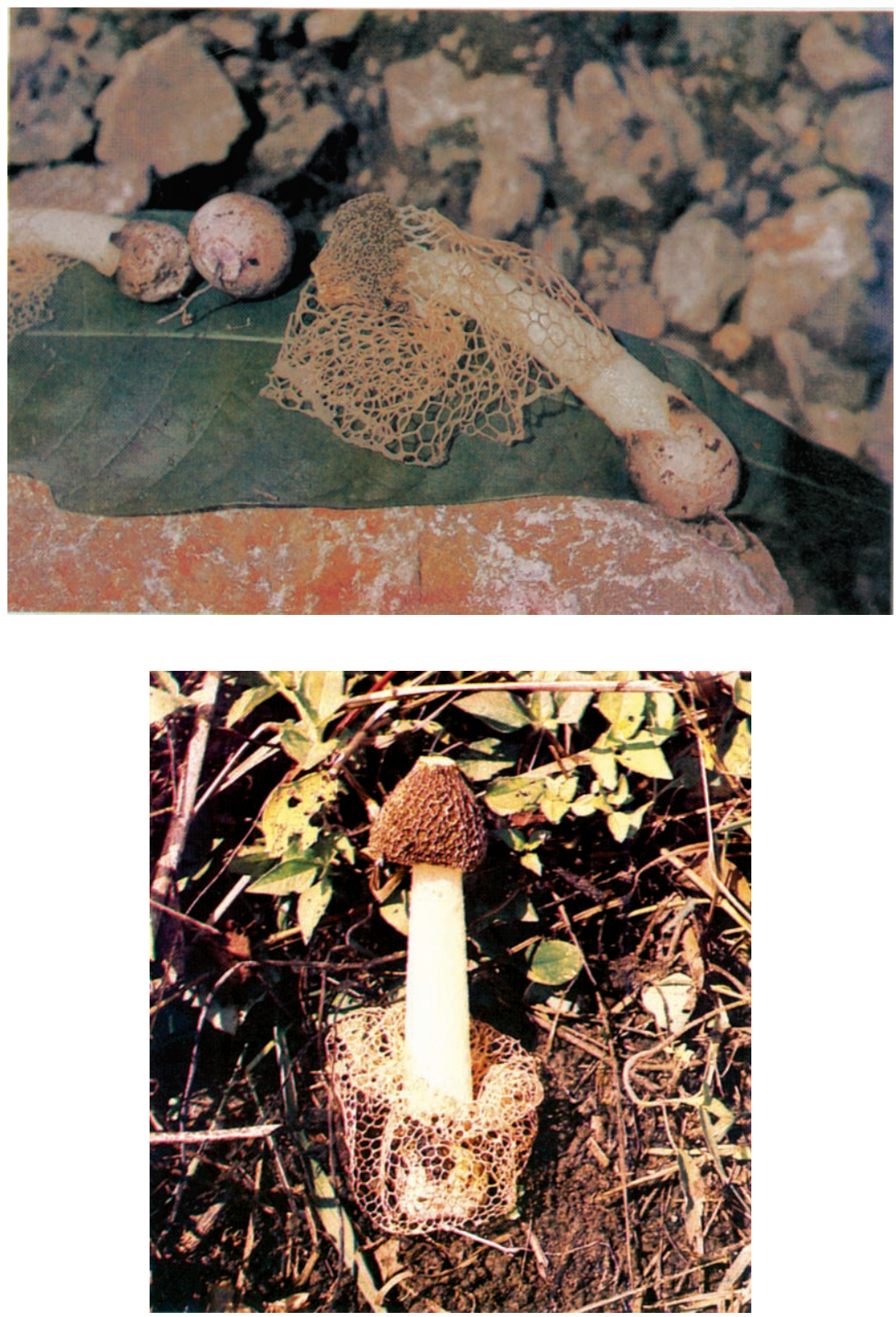

Figs. 10-11. 10. Dictyophora indusiata f. lutea, Rzedowski s.n. de Oaxaca (foto Rzedowski) (nótese el peridio de color rosa). 11. D. indusiata f. rosea, Guzmán 15713-B (foto Guzmán) de Oaxaca, con el indusio desprendido. 
Guzmán, et al.: Especies de Dictyophora en México y su Distribución en América Latina

\section{LITERATURA CITADA}

Acosta, S. y G. Guzmán. 1984. Los hongos conocidos en el Estado de Zacatecas. Bol. Soc. Mex. Mic. 19: 125-158.

Campos, M. 1987. Dictyophora indusiata (Vent. ex Pers.) Desvaux. Brenesia 28 : portada a colores y 1 a. de forros.

Chacón, S. y G. Guzmán. 1984. Nuevas observaciones sobre los hongos, líquenes y mixomicetos de Chiapas. Bol. Soc. Mex. Mic. 19: 245-252.

Coker, W. C. y J. N. Couch. 1928. The Gasteromycetes of Eastern United States and Canada. Univ. North Carolina Press. Chapel Hill. 201 pp. +123 láms.

Dennis, R. W. G. 1953. Some West Indian Gasteromycetes. Kew Bull. 3: 307-328.

Dennis, R. W. G. 1970. Fungus flora of Venezuela and adjacent countries. Kew Bull. Add. Ser. III, Royal Bot. Gardens. Kew. 531 pp. +24 láms.

Dring, D. y R. Rayner. 1967. Some Gasteromycetes from Eastern Africa. Jour. East. Afr. Nat. Hist. 26: 56.

Guzmán, G. 1959. Sinopsis de los conocimientos sobre los hongos alucinógenos mexicanos. Bol. Soc. Bot. Mex. 24: 14-34.

Guzmán, G. 1973. Some distributional relationships between Mexican and United States mycofloras. Mycologia 65: 1319-1330.

Guzmán, G. 1977. Identificación de los hongos comestibles, venenosos, alucinantes y destructores de la madera. Ed. Limusa. México, D. F. 236 pp. + 218 láms.

Guzmán, G. 1986. Distribución de los hongos en la región del Caribe y zonas vecinas. Caldasia 15: 103120.

Guzmán, G. y T. Herrera. 1973. Especies de macromicetos citadas de México, IV. Gasteromicetos. Bol. Soc. Mex. Mic. 7: 105-119.

Guzmán-Dávalos, L. y G. Guzmán. 1979. Estudio ecológico comparativo entre los hongos (macromicetos) de los bosques tropicales y los de coníferas del Sureste de México. Bol. Soc. Mex. Mic. 13: 89125.

Heim, R. y R. G. Wasson. 1958. Les champignons hallucinogènes du Mexique. Ed. Mus. Nat. Hist. Nat. París. 322 pp. +36 láms.

Kobayasi, Y. 1965a. Two colour forms of Dictyophora indusiata. Jour. Jap. Bot. 40: 178-181, + lam. III.

Kobasayi, Y. 1965b. On the genus Dictyophora, especially on the East- Asiatic group. Trans. Mycol. Soc. Japan 6: 1-8, + lam. 1.

López, A., D. Martínez y J. García. 1980. Phallales conocidos del Estado de Veracruz. Bol. Soc. Mex. Mic. 14: $39-49$.

Pilát, A. 1958. Flora CSR-Gasteromycetes. Ceskoslovenské Akad. Ved. Praga. 864 pp.

Portugal, D., E. Montiel, L. López y V.M. Mora. 1985. Contribución al conocimiento de los hongos que crecen en la región de El Texcal, Estado de Morelos. Rev. Mex. Mic. 1: 401-412.

Reid, D. A. 1977. Some Gasteromycetes from Trinidad and Tobago. Kew Bull. 31: 657-689.

Smith, A. H. 1951. Puffballs and their allies in Michigan. University of Michigan Press. Ann Arbor. 131 pp. +43 láms.

Stevenson. J. A. 1975. Fungi of Puerto Rico and the American Virgin Islands. Contr. Reed Herb. 23 Baltimore. $743 \mathrm{pp}$.

Wasson, R. G. 1959. The hallucinogenic mushrooms of Mexico: an adventure in ethmomycological exploration. Trans. New York Acad. Scien. Ser. II, 21: 325-339.

Welden, A. L. y G. Guzmán. 1978. Primera lista de los hongos, líquenes y mixomicetos de las regiones de Uxpanapa, Coatzacoalcos, Los Tuxtlas, Papaloapan y Xalapa (parte de los Estados de Veracruz y Oaxaca). Bol. Soc. Mex. Mic. 12: 59-102. 